\title{
Amiloidosis asociada a diálisis en el conducto auditivo externo, presentación de un caso clínico y revisión de la literatura
}

\author{
Dialysis related amiloydosis of the external auditory canal, case report and \\ review of the literature
}

\author{
Ximena Maul F${ }^{1}$, José Tomás Andrade $\mathbf{D}^{2}$, Daniela Contreras $\mathbf{M}^{1}$, David Jofré $\mathbf{P}^{1}$.
}

\begin{abstract}
RESUMEN
La amiloidosis asociada a diálisis es una patología causada por depósito de fibrillas amiloides constituidas por la proteína beta 2 microglobulinati-5. Es una complicación seria y excepcional que ocurre en pacientes sometidos a hemodiálisis de larga data. El caso que presentamos corresponde a un paciente de 56 años en hemodiálisis hace 36 años, con amiloidosis del conducto auditivo externo bilateral. Sólo 13 casos de amiloidosis en el conducto auditivo externo, incluido éste han sido reportados en la literatura ${ }^{6-14}$. Este corresponde al tercer caso de amiloidosis asociada a diálisis del conducto auditivo externo reportado en la literatura, 10.
\end{abstract}

Palabras clave: Amiloidosis asociada a diálisis; conducto auditivo externo.

\begin{abstract}
Dialysis related amyloidosis is a disorder caused by deposition of amyloid fibrils formed by beta-2 microglobulin ${ }^{1-5}$.

It is a serious and exceptional complication in patients undergoing long-term hemodialysis. The present case involved a 56 years old man who had been on hemodialysis for 36 years with bilateral amyloidosis of the external auditory canal. Only 13 cases of amyloidosis in the ear canal, including the present case have been reported in the literature ${ }^{6-}$ 14. This is the third reported case of dialysis related amyloidosis of the ear canalo,10.
\end{abstract}

Key words: Dialysis-related amyloidosis; external auditory canal.

\section{INTRODUCCIÓN}

La amiloidosis corresponde a un grupo de condiciones que alteran la función de los órganos y tejidos debido al depósito de amiloide, una proteína fibrilar ${ }^{6}$. Esta rara enfermedad fue descrita por Rokitansky y luego Rudolph Virchow acuñó el término amiloide en 1854. Pocos casos de amiloidosis han sido reportados en el área otorrinolaringológica, siendo los lugares más descritos la laringe y la base de la lengua ${ }^{6}$. También se han descrito depósitos de amiloide en nasofaringe, cavidades paranasales y parótida ${ }^{7-15}$. No obstante las manifestaciones otológicas son extremadamente raras ${ }^{8}$. Las áreas descritas son la concha ${ }^{16,17}$ y el conducto auditivo externo $0^{6,8,9,18}$. Hasta el año 2012 había 27 casos descritos de amiloidosis en el oído externo ${ }^{9,16,19-27}$, de los cuales 12 estaban

Médico. Departamento Otorrinolaringología, Pontificia Universidad Católica de Chile.

2 Médico. Pontificia Universidad Católica de Chile. 
ubicados en el conducto auditivo externo ${ }^{6-14}$ y sólo 2 de ellos asociados a diálisis ${ }^{6,10}$ al igual que el paciente descrito en esta publicación.

La amiloidosis asociada a diálisis corresponde al depósito de beta-2 microglobulina ${ }^{2-4}$ en pacientes dializados crónicamente ${ }^{28}$.

\section{CASO CLÍNICO}

Se reporta el caso de un paciente hombre, de 56 años con antecedentes de insuficiencia renal crónica en hemodiálisis hace 36 años, riñones poliquísticos, trasplante renal con rechazo de él en tres oportunidades, osteodistrofia renal, hiperparatiroidismo terciario operado, bypass fémoro-femoral y el diagnóstico de amiloidosis asociada a diálisis realizado en el año 2009 en relación a lesiones en la lengua.

El paciente consulta con una historia de anacusia de 20 a 30 años de evolución en el oído izquierdo en relación a ototóxicos utilizados dentro de la inmunosupresión posterior al trasplante renal. Refería tinnitus permanente en este lado, sin historia de vértigo. En la anamnesis destaca una historia de hipoacusia de meses de evolución ahora en el oído derecho que aumentó dos semanas previa a su consulta y se asocia a otalgia y otorrea. Al examen físico destaca en el oído izquierdo el conducto auditivo externo obliterado por una masa exofítica que impide ver la membrana timpánica aun bajo visión endoscópica (Figura 1). En el oído derecho los hallazgos son similares y además de encuentra otorrea. Se inicia tratamiento con ciprofloxacino vía oral, gotas de ciprofloxacino y dexametasona ótico y se solicita una audiometría y una tomografía computarizada de oídos.

En la tomografía computarizada destaca el hallazgo de ambos conductos auditivos obliterados en su totalidad por un tumor de partes blandas (Figura 2) que resulta en un lumen filiforme de ambos conductos auditivos externos. En el corte coronal derecho solo es posible ver remanentes de membrana timpánica. En el corte coronal izquierdo se observa un lumen filiforme producto de un tumor lobulado en el CAE de este lado. En la porción ósea del CAE es posible ver una erosión en sacabocado probablemente en relación a la misma enfermedad. La membrana timpánica se observa indemne.

La audiometría muestra una hipoacusia sensorioneural profunda en el lado izquierdo con una discriminación de $16 \%$ concordante con el antecedente de ototoxicidad por inmunosupresores, y una hipoacusia de conducción al lado derecho con un gap de $50 \mathrm{~dB}$ y una discriminación de $96 \%$ en este lado.

Se decide extirpar la lesión del oído derecho bajo anestesia local en la consulta ambulatoria y se envía el tejido a anatomía patológica. En la Figura 3 se observa la imagen del CAE derecho aproximadamente 1 año
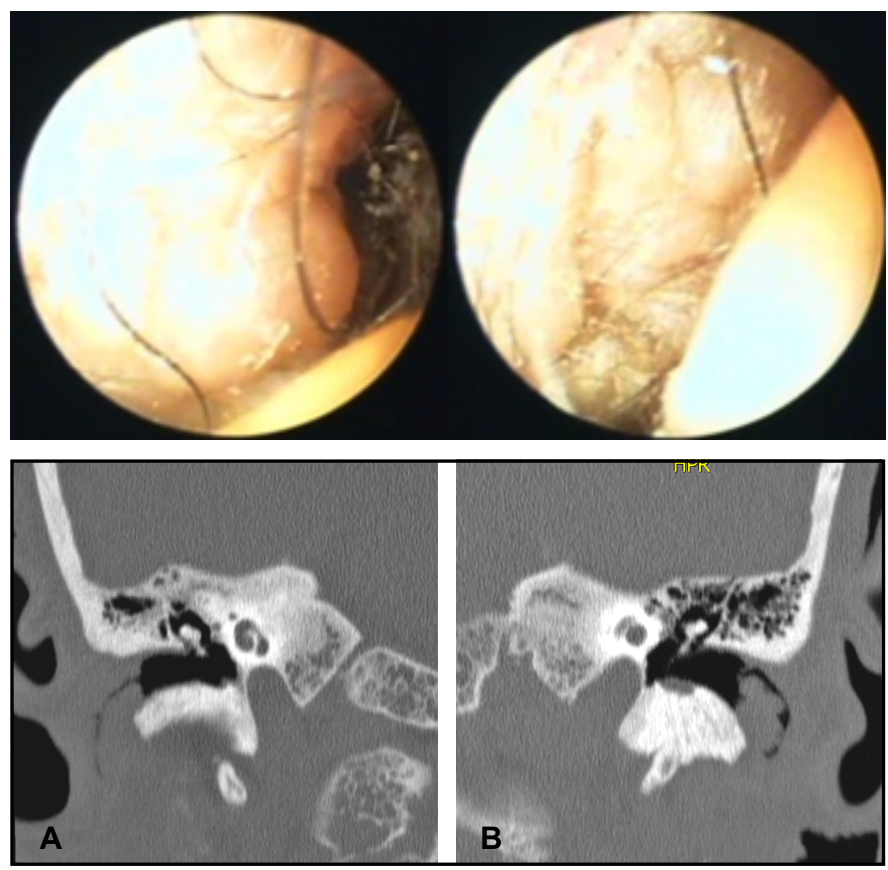

Figura 1. Tumor del conducto auditivo externo (CAE) izquierdo bajo visión endoscópica. Hallazgo similar en el CAE derecho.

Figura 2a y 2b. TC Oídos (2a corte coronal derecho y $2 \mathrm{~b}$ izquierdo). 
Figura 3. Control 1 año posterior a la resección parcial de la lesión del conducto auditivo derecho. A la derecha se observa una perforación holotimpánica que permite ver el oído medio.

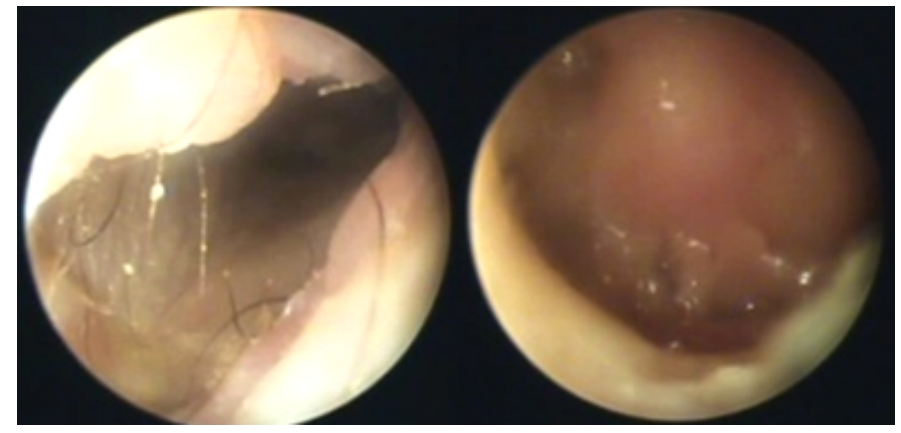

Figura 4 a y 4b. Corte histológico, tinción hematoxilina eosina (4a: Se observa epidermis atrófica y depósito dérmico de material eosinófilo, amorfo, con telangiectasias; $\mathrm{HE}$ 200X y 4b: Material amiloideo amorfo. HE, $400 X)$.

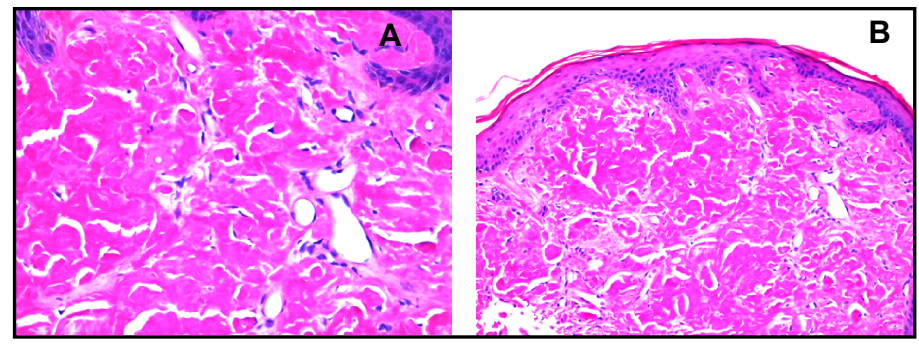

posterior a la resección, con lesión residual o recidiva. Además se pesquisa una gran perforación holotimpánica a derecha, este antecedente de otitis media crónica no se conocía previamente.

La anatomía patológica del tejido obtenido del CAE revela restos de piel con depósito de amiloide y se observa en la tinción de rojo congo, una birrefringencia verde amarillenta bajo la luz polarizada. En la Figura 4 se observan 2 cortes histológicos de la lesión amiloidea con tinción hematoxilina eosina.

Este caso es compatible con el diagnóstico de amiloidosis del conducto auditivo externo bilateral y por el antecedente del paciente, es altamente probable que corresponda a amiloidosis del CAE asociada a diálisis.

\section{REVISIÓN DE LA LITERATURA}

En cuanto a la amiloidosis, esta es una enfermedad de causa desconocida que se produce por el depósito extracelular de amiloide, una sustancia fribrilar de origen proteico y estructura beta plegada ${ }^{29}$. Es poco frecuente con una incidencia de 8 por 1.000 .000 de habitantes. En la histología nos encontramos en la tinción de hematoxilina eosina, material amorfo eosinófilo y en la tinción de rojo congo es posible encontrar una birrefringencia verde manzana lo cual es patognomónico del diagnóstico de amiloidosis.

En cuanto a su clasificación, ésta ha variado a lo largo del tiempo. Actualmente es posible hablar de enfermedad amiloidea primaria, secundaria, familiar, familiar renal exclusiva, asociada a diálisis y órgano específica. Todas estas a su vez pueden ser sistémicas o localizadas dependiendo si existe compromiso de 10 más órganos ${ }^{30}$. La enfermedad amiloidea primaria, se produce por depósito de cadenas de inmunoglobulinas livianas o pesadas y puede coexistir con el diagnóstico de mieloma múltiple, gamapatía monoclonal de significado incierto o macroglobulinemia de Waldestrom. La enfermedad amiloidea secundaria se debe al depósito de proteína amiloidea A y se produce por infecciones 0 estados inflamatorios crónicos como la tuberculosis, espondiloartropatías, artritis reumatoídea o drogas entre otros. En la enfermedad amiloidea familiar la proteína alterada es la transtiretina, en la familiar renal exclusiva lo es el fibrinógeno, en la enfermedad amiloidea asociada a diálisis la proteína de depósito es la beta 2 microglobulina. En cuanto a la enfermedad amiloidea órgano específica, el sistema nervioso central es lo más estudiado con la enfermedad de Alzheimer donde la proteína de depósito es la proteína beta amiloide ${ }^{30}$.

En cuanto a la amoiloidosis asociada a diálisis, como se mencionó anteriormente ésta se debe al depósito de beta 2 microglobulina ${ }^{29}$. Esta proteína es producida por los leucocitos y es parte del complejo mayor de histocompatibilidad de la superficie celular. Se cataboliza en el túbulo proximal renal por lo 
que se acumula en pacientes con falla renal crónica y las membranas de diálisis no la depuran por lo que llega hasta 30 a 60 veces su valor normal en pacientes insuficientes renales en hemodiálisis. En general los pacientes que la padecen son pacientes con más de 10 años en hemodiálisis.

En el contexto de la amiloidosis en otorrinolaringología, la amiloidosis en el conducto auditivo externo es extremadamente rara. Lo más frecuente en la especialidad es encontrar el compromiso de laringe en primer lugar, luego base de lengua y luego faringe. Aunque también se ha descrito compromiso de cavidades paranasales, tráquea, entre otros.

\section{DISCUSIÓN}

Hasta el año 2011 solamente había 12 casos en el mundo descritos de amiloidosis en el conducto auditivo externo de los cuales sólo 2 habían sido amiloidosis asociada a diálisis como el presente caso. La Tabla 1 muestra un resumen de los tres casos reportados hasta la fecha de amiloidosis asociada a diálisis del CAE. Lo más frecuente es encontrar el compromiso bilateral de los oídos debido a enfermedad amiloidea ${ }^{6}$.

En ambos casos previamente reportados el tratamiento fue la resección de las lesiones, la anatomía patológica confirmó el diagnóstico y en el seguimiento no hubo recurrencia de las lesiones ni crecimiento al año de seguimiento. El antecedente de hemodiálisis de larga data es un factor común en los 3 casos de amiloidosis del CAE por diálisis reportados lo que es concordante con estudios posmortem que han encontrado depósito de amiloide en $21 \%$ de los pacientes dializados por menos de dos años, $50 \%$ entre 4 a
7 años, 90\% entre 7 y 13 años y 100\% en los dializados por más de 13 años ${ }^{31}$.

En cuanto al tratamiento de la amiloidosis asociada a diálisis, lo principal sería la prevención a través de la mejoría de las membranas de diálisis ${ }^{6}$. Se han reportado columnas adsorbentes de beta 2 microglobulina que podrían ayudar a filtar esta proteína en la hemodiálisis, al igual que membranas más biocompatibles, todas estas alternativas son de alto costo y no disponibles a nivel nacional ${ }^{32}$.

Los casos localizados de amiloidosis son de buen pronóstico con la resección de las lesiones y luego seguimiento cercano, mientras que los casos de enfermedad sistémica son hasta la fecha de manejo sintomático y con tratamientos biológicos aún en fase de investigación ${ }^{6}$.

Este caso nos parece de gran interés por 10 infrecuente del diagnóstico en nuestra especialidad y para tenerlo presente dentro de los diagnósticos diferenciales de tumores en el conducto auditivo externo.

\section{BIBLIOGRAFÍA}

1. Fenves AZ, Emmett M, White MG, et al. Carpal tunnel syndrome with cystic bone lesions secondary to amyloidosis in chronic hemodialysis patients. Am J Kidney Dis 1986; 7: 130.

2. Косн KM. Dialysis-related amyloidosis. Kidney Int 1992; 41: 1416.

3. Gejyo F, Odani S, Yamada T, et Al. Beta 2-microglobulin: a new form of amyloid protein associated with chronic hemodialysis. Kidney Int 1986; 30: 385.

4. Winchester JF, Salsberg JA, Levin NW. Beta-2 microglobulin in ESRD: an in-depth review. $A d v$ Ren Replace Ther 2003; 10: 279.

Tabla 1. Resumen de casos reportados hasta la fecha de amoiloidosis en el CAE asociada a diálisis

\begin{tabular}{|c|c|c|c|}
\hline Casos reportados & $2002^{13}$ & $2011^{6}$ & $2012\left({ }^{*}\right.$ presente caso) \\
\hline Antecedentes & $\begin{array}{l}\text { Mujer 46a, IRC en HD } \\
\text { hace } 20 \text { a. }\end{array}$ & $\begin{array}{l}\text { Hombre } 56 \mathrm{a} \text {, IRC en HD } \\
\text { hace } 12^{\mathrm{a}} \text {. }\end{array}$ & $\begin{array}{l}\text { Hombre 56a, IRC en HD } \\
\text { hace } 36 \mathrm{a} \text {. }\end{array}$ \\
\hline MC & Hipoacusia bilateral & Tumor en CAE & Hipoacusia bilateral \\
\hline Examen físico & Masas color café CAE & Obliteración CAE por tumor & Obliteración CAE bilateral por tumor \\
\hline Audiometría & H conducción 30 dB & H mixta $60 \mathrm{~dB}$ & $\begin{array}{l}\text { H conducción OD 50dB, } \\
\text { HSN profunda Ol }\end{array}$ \\
\hline TC de oído & Compromiso ambos CAE & $\begin{array}{l}\text { Compromiso CAE D } D^{0} \text {, MT, } \\
\text { mastoides y hueso CAE }\end{array}$ & $\begin{array}{l}\text { Compromiso CAE bilateral. } \\
\text { Erosion ósea del CAE }\end{array}$ \\
\hline Tratamiento & Excisión parcial bajo A. Local & Resección A. General_endo y retro & Excisión parcial bajo A. local \\
\hline A patológica & Rojo congo (t), B2-MG (t). & Tinción B2 MG + & Rojo congo $(+)$ \\
\hline Seguimiento & Sin crecimiento al año & Sin recurrencia al año & Lesión residual al año \\
\hline
\end{tabular}


5. Dember LM, Jaber BL. Dialysis-related amyloidosis: late finding or hidden epidemic? Semin Dial 2006; 19: 105.

6. Kazuharu Yamazaki et al. A case of hemodialysisassociated amyloidosis localized to the external auditory canal. Auris Nasus Larynx 2011; 38: 295-9.

7. Zundel RS, PyLe GM, Voytovich M. Head and neck manifestations of amyloidosis. Otolaryngol Head Neck Surg 1999; 120: 553-7.

8. Iván El-SAYED, Nicolas Y. Busaba, and William C. Faquin. Otologic Manifestations of Amyloidosis. Otology \& Neurotology 2002; 23: 158-9 .

9. Panarese A, Roland NJ, Green B. Primary amyloidosis of the external auditory canal: Case report. J Laryngol Otol 1994; 108: 49-50.

10. Ashimori N, Hayashi Y, Mineta H, Hoshino T. Dialysis-related amyloidosis of the external auditory canal. Am J Otol 2002; 23: 374-7.

11. Noojin RO, ArRington TS. Unusual cutaneous findings in primary systemic amyloidosis. Arch Dermatol 1965; 92: 157-9.

12. Gheriani H, Tewary R, O'Sullivan TJ. Amyloidosis of the external auditory canal and middle ear: unusual ear tumor. Ear Nose Throat J 2007; 86: 92-3.

13. Alvarez-Ruiz SB, Pérez-Gala S, Aragues M, Fraga J, García-Diez A. Unusual clinical presentation of amyloidosis: bilateral stenosis of the external auditory canal, hoarseness and a rapid course of cutaneous lesions. Int J Dermatol 2007; 46: 503-4.

14. Ashimori N, HaYaSH Y, Hoshino T. Two cases of amyloidosis of the externalauditory canals. Jibi Rinsyo 2003; 96: 1049-54.

15. Nandapalan V, Jones TM, Morar P, et al. Localized amyloidosis of the parotid gland: A case report and review of the localized amyloidosis of the head and neck. Head Neck 1998; 20: 73-8.

16. Hicks BC, Weber PJ, Hashimoto K, et al. Primary cutaneous amyloidosis of the auricular concha. J Am Acad Dermatol 1988; 18: 19-25.

17. Bakos L, Weissbluth ML, Pires AK, et al. Primary amyloidosis of the concha. J Am Acad Dermatol 1989; 20: 524-5.

18. Mozos A, et AL. Amiloidosis del canal auditivo externo. Acta Otorrinolaringol Esp 2010; 62: 392-4.

19. Echevarría de Martínez J, Nanita de Esteve F, Brea Z.
Liquen amiloide de pabellon auricular, reporte de ocho casos. Rev Domin Dermatol 1980; 14: 59.

20. Dupre A. Hyalinose micro-papuleuse de laconque des oreilles avec elimination transepidermique,cas pour diagnostic. Presentationde posters. In Reunion de la Societe Francaise de Dermatologie. Toulose: S'eanced'histopathologie cutanee, 1981.

21. MASCARO J. Liquen amiloide del pabellonauricular. In VIle Reunion Internationale de Dermatologie. Barcelona, 1982.

22. Weissbluth ML, Muller LFB PA, Bakos L.Lichen amiloide com lesoes auriculars. An Bras Dermatol 1987; 62: 49.

23. Barnadas $M$, Pérez $M$, Esquius J, Curell $R$, de Moragas JM. Papules in the auricular concha: lichen amyloidosus in a case of biphasic amyloidosis. Dermatologica 1990; 181: 149.

24. Mahalingam M, Palko M, Steinberg-Benjes L, GoLDBERG L. Amyloidosis of the auricular concha: an uncommon variantof localized cutaneous amyloidosis. Am J Dermatopathol2002; 24: 447.

25. ShimauchI T, ShIN JH, TokURA Y. Primary cutaneous amyloidosis of the auricular concha: case report and review of published work. J Dermato/2006; 33: 128.

26. ERRol C. Lichen amyloidosis of the auricular concha: report of two cases and review of the literature. Dermatol Online J 2006; 12 : 1.

27. Neff AG, McCuin JB, Mutasim DF. Papular amyloidosis limited to the ears. J Am Acad Dermatol 2010; 62: 1078.

28. Westermark P. Classification of amyloid fibril proteins and their precursors: An ongoing discussion. Int J Exp Clin Invest 1997; 4: 216-8.

29. Pérez S. Amiloidosis. Rev Chil Reumatol 2008; 24(4): 200-5.

30. Gertz MA, Rajkumar SV. Primary Systemic Amiloydosis. Curr Treat Opt Oncol 2002; 3: 261-71.

31. Jadoul M, Garbar C, Noel H, et al. Histological prevalence of beta 2-microglobulin amyloidosis in hemodialysis: a prospective post-mortem study. Kidney Int 1997; 51: 1928-32.

32. Yamamoto Y, Hirawa N, Yamaguch $S$ et al. Long-term efficacy and safety of the small-sized B2microbglobulin adsorption column for dialysis-related amyloidosis. Ther Apher Dial 2011; 15(5): 466-74. 